\title{
The Medial Prefrontal Cortex, Nucleus Accumbens, Basolateral Amygdala, and Hippocampus Regulate the Amelioration of Environmental Enrichment and Cue in Fear Behavior in the Animal Model of PTSD
}

\author{
Ying Hao Yu, ${ }^{1,2}$ Yeou San Lim, ${ }^{1}$ Chen Yin Ou, ${ }^{1}$ Kai Chieh Chang, ${ }^{1}$ Arthur C. Tsai, ${ }^{3}$ \\ Fang Chih Chang, ${ }^{1}$ and Andrew Chih Wei Huang ${ }^{1}{ }^{1}$ \\ ${ }^{1}$ Department of Psychology, Fo Guang University, Yilan County 26247, Taiwan \\ ${ }^{2}$ Department of Biotechnology and Animal Science, National Ilan University, Yilan County 26247, Taiwan \\ ${ }^{3}$ Institute of Statistical Science, Academia Sinica, Taipei, Taiwan \\ Correspondence should be addressed to Andrew Chih Wei Huang; acwhuang@gmail.com
}

Received 27 August 2021; Revised 7 January 2022; Accepted 27 January 2022; Published 7 February 2022

Academic Editor: Enzo Emanuele

Copyright (c) 2022 Ying Hao Yu et al. This is an open access article distributed under the Creative Commons Attribution License, which permits unrestricted use, distribution, and reproduction in any medium, provided the original work is properly cited.

\begin{abstract}
A growing body of evidence showed that environmental enrichment (EE) ameliorated footshock-induced fear behavior of posttraumatic stress disorder (PTSD). However, no research comprehensively tested the effect of EE, cue, and the combination of EE and cue in footshock-induced fear behavior of PTSD symptoms. The present study addressed this issue and examined whether the medial prefrontal cortex (mPFC, including the cingulate cortex 1 (Cg1), prelimbic cortex (PrL), and infralimbic cortex (IL)), the nucleus accumbens (NAc), the basolateral amygdala (BLA), and the hippocampus (e.g., CA1, CA3, and dentate gyrus (DG)) regulated the amelioration of the $\mathrm{EE}$, cue, or the combination of $\mathrm{EE}$ and cue. The results showed that $\mathrm{EE}$ or cue could reduce fear behavior. The combination of EE and cue revealed a stronger decrease in fear behavior. The cue stimulus may play an occasion setting or a conditioned stimulus to modulate the reduction in fear behavior induced by footshock. Regarding the reduction of the EE in fear behavior, the Cg1 and IL of the mPFC and the NAc upregulated the cFos expression; however, the BLA downregulated the c-Fos expression. The mPFC (i.e., the Cg1, PrL, and IL) and the hippocampus (i.e., the CA1, CA3, and DG) downregulated the c-Fos expression in the suppression of the cue in fear behavior. The interaction of EE and cue in reduction of fear behavior occurred in the Cg1 and NAc for the c-Fos expression. The data of c-Fos mRNA were similar to the findings of the c-Fos protein expression. These findings related to the EE and cue modulations in fear behavior may develop a novel nonpharmacological treatment in PTSD.
\end{abstract}

\section{Introduction}

Posttraumatic stress disorder (PTSD) is a complicated mental illness [1]. PTSD individuals often encounter a variety of psychiatric symptoms that include feeling fear, helplessness, horror, increased arousal, autonomic hyperarousal activities, fear sensitization, serious startle responses, hypervigilance, insomnia, irritability, and impaired concentration [2-4]. Concerning the numerous symptoms of PTSD, the fear symptom through classical conditioning to form fear memory in the animal model of PTSD effectively simulated the PTSD's human symptoms [5]. Moreover, PTSD's fear symptom is crucial to the etiology of PTSD, and it is highly preserved in the process of evolution cross-species [5].

In light of the previous studies, environmental enrichment (EE) exposures can effectively reduce the PTSD symptoms in the animal model [6-11]. For example, EE reduced anxiety due to differentially activating the serotonin system and neuropeptide system in the PTSD animal model [11]. Chronic $\mathrm{EE}$ exposures reduced neonatal isolation-induced contextual freezing; however, chronic EE exposures did not affect single prolonged stress-related to anxiety behaviors or analgesia 
[9]. Acute EE exposures facilitated fear extinction through neuropeptide Y-Y1 receptor modulation in the hippocampus [10]. EE enhanced anxiolytic effect induced by deep-brain stimulation in the medial prefrontal cortex (mPFC) in the PTSD animal model [6]. Traumatic stress caused the reduction in the volume of the hippocampus and central amygdala, and $\mathrm{EE}$ was able to ameliorate trauma-induced volume decreases of the hippocampus resulted in behavioral, morphological, and molecular changes [8]. EE facilitated the negative feedback of the hypothalamus-pituitary-adrenal gland (HPA) axis-related stress system and then improved abnormal behaviors through decreasing the glucocorticoid receptors in the hypothalamus and hippocampus [7]. Therefore, EE is a nonpharmacological treatment in reducing PTSD symptoms at behavioral, neuronal, and molecular levels.

The EE manipulation combined exposure of sensory, physical, cognitive, and social stimuli in the contextual surroundings for individuals, and the EE simulated a contextual environment. Animals exposed the entire components of sensory, physical, social, and cognitive stimuli [12-14]. In contrast to the EE stimuli, the cue is a single component or stimulus of the contextual stimuli [15]. Based on the previous evidence, the different category of fear conditioning is controlled by the different neural substrates: the hippocampus is contributable to the context-induced fear conditioning $[15,16]$, whereas the amygdala modulates the cue-induced fear conditioning $[17,18]$. The hippocampus plays a function in configural memory and the encoding process of contextual stimulus [19]. The amygdala is essential to regulate the negative valence of emotion and negative emotional processes [20]. Recently, accumulated studies have clarified the roles of the mPFC, amygdala, and hippocampus in the fear conditioning of the PTSD symptoms after encountering a traumatic stress event [21-24]. The individual who experienced traumatic events was revealed to decrease the volumes of the hippocampus and anterior cingulate cortex; however, it increased the hyperactivity of the amygdala; moreover, the mPFC showed dysfunction that cannot inhibit the activity of the amygdala [21]. The hippocampus contributed to spatial learning and declarative memory; moreover, the hippocampus was projected from the amygdala, and thereby, the hippocampus-related functions, including spatial learning and memory, were regulated by the amygdala's activity [25]. Alternatively, little research has demonstrated that the nucleus accumbens (NAc) might be involved in the PTSD symptoms associated with stress behaviors [26, 27]. Therefore, the study examined whether the mPFC, amygdala, hippocampus, and NAc mediated fear behavior of PTSD symptoms.

Altogether, some critical issues should be concerned: (a) Whether EE decreased fear symptoms of PTSD. (b) Whether a stimulus cue can reduce PTSD fear symptoms. (c) Using the c-Fos labeling and the assessment of c-Fos mRNA expression with the qRT-PCR methods, whether the subareas of mPFC (e.g., the Cg1, PrL, and IL), the subregions of the hippocampus (e.g., the CA1, CA3, and DG), the NAc, and BLA were involved in the amelioration of $\mathrm{EE}$, cue, or the EE and cue combination in footshock-induced fear behavior of the PTSD symptom.

\section{Methods and Materials}

2.1. Animals. Fifty-six male wild-type C57BL/6 mice (approximate 25 35 $\mathrm{g}$ at the beginning of the experiments) were bought from National Laboratory for Animal Breeding and Research Center, Taipei, Taiwan. All mice were randomly assigned into no environmental enrichment (no EE; i.e., standard housing) and EE cages. The mice of no EE and $\mathrm{EE}$ groups were raised with another two mice in a colony room with constant temperature (approximately $23 \pm$ $2^{\circ} \mathrm{C}$ ) and light-dark cycle (light on 6:00-18:00). The EE cage is a plastic surrounding shell box, which is $33.5 \mathrm{~cm}$ long $\times 25.0 \mathrm{~cm}$ wide $\times 28.8 \mathrm{~cm}$ high. The standard cage is a plastic box, and its surrounding size is $30.0 \mathrm{~cm}$ long $\times 18.8$ $\mathrm{cm}$ wide $\times 13.5 \mathrm{~cm}$ high. Food and water were provided ad libitum. The experiments were performed in compliance with the American Psychological Association ethical standards for the treatment of animals. A description of the treatment details was submitted and received approval from the Institutional Animal Care and Use Committee (IACUC) of Fo Guang University. Every effort was made to minimize the animals' suffering and the number of animals used.

\subsection{Apparatus}

2.2.1. Inescapable Footshock. The inescapable footshock apparatus is a box composed of a plastic surrounding shell measuring $60 \mathrm{~cm} \times 60 \mathrm{~cm} \times 72 \mathrm{~cm}$ high. The apparatus floor is composed of metal grids $(0.3 \mathrm{~cm}$ diameter at $0.7 \mathrm{~cm}$ grid intervals). On the footshock procedure, mice were placed in the footshock apparatus for $2 \mathrm{~min}$. Then, mice were given a $2 \mathrm{~mA}$ footshock (duration, 10 seconds), and the other mice received no footshock at their home cage [28].

2.3. Behavioral Procedure. All mice received an adaptation regimen for ad libitum food and water for seven days in the adaptation phase. After that, all mice were dependent on their assigned groups, and they were, respectively, raised in a mixed environment, including any combinations of no $\mathrm{EE}, \mathrm{EE}$, no cue, and cue until the end of the experiment (day 0 -day 20). On day 17 , all mice were given a single footshock ( $2 \mathrm{~mA}, 10$ seconds) in the footshock apparatus. On days $18-20$, all mice received the situational reminder procedure. Note, the current rate of footshock ( $2 \mathrm{~mA}, 10$ seconds) and three freezing-times in situational reminders were determined by our previous studies [22, 29]. The mice were placed in the footshock apparatus for $2 \mathrm{~min}$ without footshock. One hundred twenty minutes after completing the last behavioral test, the immunohistochemical staining and the qRT-PCR method were, respectively, performed to measure c-Fos protein and c-Fos mRNA expression in the selected brain areas (see Figure 1).

All of the mice were assigned into $\mathrm{EE}$ or cue environments into four groups: no EE/no Cue, no EE/cue, EE/no cue, and EE/cue groups ( $n=6$, per group). Regarding the housing condition, the mice in the no $\mathrm{EE} / \mathrm{No}$ cue group were given no $\mathrm{EE}$ and no cue in the standard cage. The no EE/cue group mice were given no $\mathrm{EE}$ with a cue stimulus in the standard cage. The mice in the $\mathrm{EE} /$ no cue group were given an EE procedure without a cue stimulus. The mice in the EE/ 


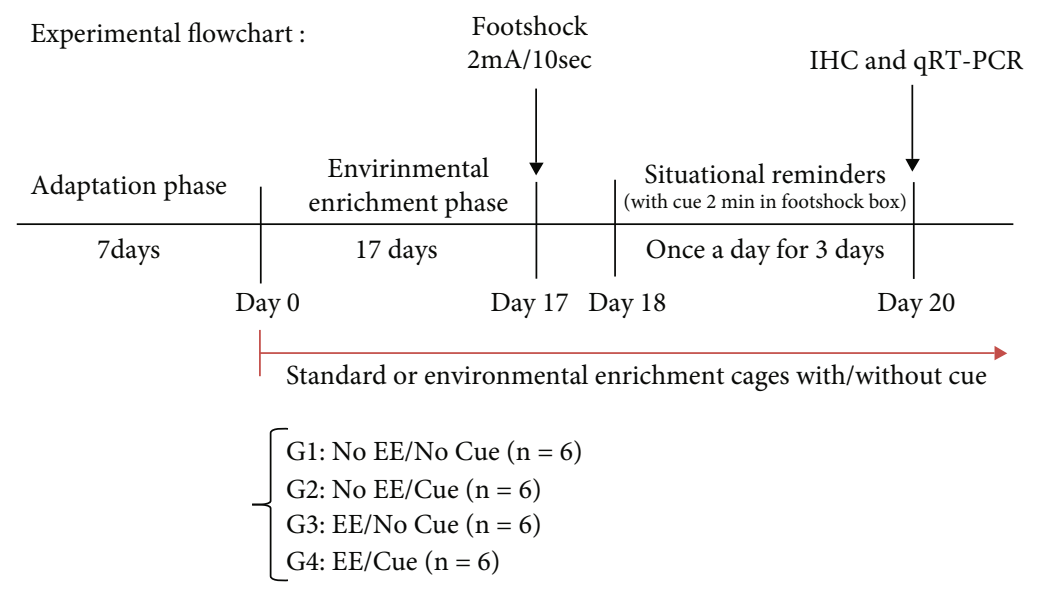

FIGURE 1: Overview of the experimental procedures. The behavioral processes were shown in the adaptation, EE phase, footshock treatment, and situational reminder phases. On days 0-17, the mice in the EE groups were subjected to the EE procedure. These mice received the EE procedure at the end of the experiment. However, the mice in the no EE groups were housed in the standard cage. On day 17 , all mice were placed in the footshock box for $2 \mathrm{~min}$, and then the mice were subjected to the footshock treatment $(2 \mathrm{~mA}, 10 \mathrm{~seconds})$. On days $18-20$, all mice have given a situational reminder procedure that the mice were placed in the footshock box for 2 min with the cue ball but not any footshock. One hundred twenty minutes after the last behavioral test, the immunohistochemical staining with the c-Fos protein expression on day 20. All mice were assigned to the no EE/no cue, no EE/cue, EE/no cue, and EE/cue groups ( $n=6$, per group). EE: environmental enrichment.

cue group were given an EE procedure with a cue stimulus. The EE cage included two large wooden blocks, a shelter, a retreat, a tunnel, crow ball, motor running wheel, and bone toys, and the EE procedure was followed the previous study [30]. The cue stimulus was designed for the mice exposed to a plastic round ball (diameter is $3 \mathrm{~cm}$ ) in the EE or standard cages for 24 hours. This cue stimulus, a white round ball, is different in size and texture from the crow ball of the EE cage.

2.4. Immunohistochemical Staining. Mice were sacrificed by sodium pentobarbital overdose after completing behavioral tests for $120 \mathrm{~min}$ (i.e., labeling IHC c-Fos for the best expression). When completely unresponsive, mice were perfused with $0.9 \% \mathrm{NaCl}$ followed by $4 \%$ paraformaldehyde in $0.1 \mathrm{M}$ sodium phosphate-buffered saline (PBS). The brain tissues were dissected, blocked, postfixed for 3 days, and transferred to $30 \%$ sucrose for cryoprotection for 2 days until the specimens sink to the bottom of the solution. Forty-micron coronal sections were cut on freezing using a sliding microtome. All brain slices were processed by c-Fos immunoreactivity staining. Free-floating brain slices were washed once for 10 mins in $0.1 \mathrm{M}$ PBS, permeabilized in $3 \% \mathrm{H}_{2} \mathrm{O}_{2}$ for 1 hour, washed three times in PBS for $10 \mathrm{mins}$, and then soaked in normal goat serum for 1 hour. After washing with PBS once for $10 \mathrm{mins}$, the slices were incubated overnight with the specific first antibody (i.e., sheep anti-c-Fos primary antibody at a dilution of 1:500). This antibody was raised against a peptide sequence to enable crossreaction with other proteins for detecting c-Fos immunoreactivity. The slices were then washed once with PBS for 10 mins, and then, these slices were incubated in a second antibody (i.e., biotinylated anti-sheep secondary antibody at a dilution of 1:500) for 2 hours. Ten minutes with PBS washing later, the bound secondary antibody was amplified using the Vector Elite ABC kit.

The positive expression nucleus of neurons was quantified for the selected brain areas. Counting was performed visually at $\times 20$ magnification by a researcher blinded to the condition of each mouse. Every third slice of the brain tissue was selected into an available counted section. The software ImageJ was used to count the amounts of c-Fos-positive neurons [31].

2.5. Real-Time Quantitative PCR of c-Fos. Based on the manufacturer's instructions, total RNA was extracted with TRIzol Reagent (Invitrogen, Carlsbad, CA, USA). For cDNA synthesis, total RNA was applied with random hexamers. Later, the reverse-transcription PCR was the amplification of $c$-fos. For the amplification, it was initiated with a pair of $c$-fos primers (forward: $5^{\prime}$-TCCACTGCCTGGGACA GAA-3'; reverse: $5^{\prime}$-CGCAGCGATCTTCATCAAAC- $3^{\prime}$ ) with a volume of $20 \mu \mathrm{l}$. For undergoing the process, it was a denaturation stage at $95^{\circ} \mathrm{C}$ for 10 minutes. After that, 28 cycles of denaturation were conducted at $95^{\circ} \mathrm{C}$ for one minute. The primer was annealing at $55^{\circ} \mathrm{C}$ for 30 seconds and extension at $72^{\circ} \mathrm{C}$ for 45 seconds. The cycling steps were completed, and the final extension was at $72^{\circ} \mathrm{C}$ for five minutes. The reactions were repeated three times and were performed in an ABI PRISM 7500 Sequence Detection System (Applied Biosystems, Thermo Fisher Scientific, USA). The mean expression levels of the housekeeping gene were with a pair of beta-actin primers (forward: $5^{\prime}$ CAACTT GATGTATGAAGGCTTTGGT-3'; reverse: $5^{\prime}$-ACTTTT ATTGGTCTCAAGTCAGTGTACAG-3 ${ }^{\prime}$ ). It was used as the internal control to normalize the variability of $c$-fos expression levels. Relative changes in the gene expression were measured using the $2^{-\Delta \Delta \mathrm{CT}}$ method [32]. 
TABLE 1: Mean ( \pm SEM) freezing time (sec.) was analyzed by a $2 \times 2 \times 3$ three-way mixed analysis of variance (ANOVA) (environmental enrichment vs. cue vs. session).

\begin{tabular}{lr}
\hline $2 \times 3 \times 2$ three-way mixed ANOVA & $F_{1,20}=70.73, p<0.05^{*}$ \\
Environmental enrichment & $F_{1,20}=14.14, p<0.05^{*}$ \\
\hline Cue & $F_{2,40}=27.95, p<0.05^{*}$ \\
Session & $F_{1,20}=3.25, p>0.05$ \\
Environmental enrichment $\times$ cue & $F_{2,40}=2.11, p>0.05$ \\
Environmental enrichment $\times$ session & $F_{2,40}=0.28, p>0.05$ \\
Cue $\times$ session & $F_{2,40}=0.89, p>0.05$ \\
Environmental enrichment $\times$ cue $\times$ session & $=0.05$ \\
\hline
\end{tabular}

${ }^{*} p<0.05$, significant difference.

2.6. Statistical Analysis. A three-way mixed (environmental enrichment vs. cue vs. session) analysis of variance (ANOVA) was performed for the freezing time. One-way ANOVA was conducted for the total freezing time in the main effect of environmental enrichment, cue, and situational reminders. Furthermore, two-way mixed (group vs. session) ANOVA was performed for the freezing time over sessions 1-3 in situational reminders and total freezing time among the no EE/no cue, no EE/cue, EE/no cue, and EE/Cue groups. When appropriate, the post hoc with Tukey's Honest Significant Difference (HSD) test was performed.

Regarding the examination of the c-Fos protein expression and c-Fos mRNA expression, two-way ANOVA analysis was conducted, and one-way ANOVA analysis was performed. For c-Fos protein expression analysis, the selected brain areas were determined, including Cg1, PrL, IL, NAc, BLA, CA1, CA2, and DG. For c-Fos mRNA expression analysis, the mPFC, NAc, amygdala, and hippocampus were determined. When appropriate, the post hoc with Tukey's HSD test was performed. ${ }^{*} p<0.05$ was considered statistically significant compared to the no EE/no cue group. $\# p<0.05$ was considered statistically significant compared to the no EE/cue group. $\$ p<0.05$ was considered statistically significant compared to the EE/no cue group.

\section{Results}

3.1. PTSD Behavioral Tests. To examine the effect of environmental enrichment and cue in freezing behavior of the PTSD animal model, a $2 \times 2 \times 3$ three-way mixed ANOVA analysis indicated that significant differences occurred in the factor of $\operatorname{EE~}(F(1,20)=70.73, p<0.05)$, cue $(F(1,20)=14.14, p<0.05)$, and session $(F(2,40)=27.95$, $p<0.05)$. Nonsignificant differences occurred in the interaction of $\mathrm{EE}$ and cue $(F(1,20)=3.24, p>0.05)$, the interaction of $\mathrm{EE}$ and session $(F(2,40)=2.11, p>0.05)$, the interaction of cue and session $(F(2,40)=0.28, p>0.05)$, and the interaction of EE, cue, and session $(F(2,40)=0.89$ $, p>0.05)$. The results indicated that EE, cue, and session had significant differences (Table 1). Furthermore, one-way ANOVA analysis was performed. The results showed that the main effect of EE was significantly decreased in total freezing time $(F(1,22)=41.62, p<0.05$; Figure 2$)$. The main effect of cue was seemingly decreased in total freezing time $(F(1,22)=3.21, p=0.08$; Figure 3$)$. The main effect of session was significantly decreased from session 1 to session $3(F(2,46)=27.61, p<0.05$; Figure 4$)$. The post hoc Tukey's HSD appeared that total freezing time of session 2 and 3 was significantly decreased when compared to session $1(p<0.05)$. The total freezing time of session 3 was significantly decreased than that of session $2(p<0.05)$. In summary, total freezing time was significantly decreased as manipulations of EE, cue, and sessions.

Alternatively, a $4 \times 3$ mixed two-way ANOVA analysis was conducted for freezing behavior. The results showed that significant differences occurred in the factor of group $(F(3,20)=29.37, p<0.05)$ and session $(F(2,40)=27.95$, $p<0.05)$. However, there was a nonsignificant difference in the interaction of group and session $(F(6,40)=1.09, p>$ 0.05). Post hoc with Tukey's HSD tests showed that the freezing time in the no EE/cue, EE/no cue, and EE/cue groups was seemingly decreased compared to the no EE/no cue group in sessions 1 and $2(p<0.05)$. In session 3 , the freezing time in the no EE/cue, EE/no cue, and EE/cue groups was significantly decreased than that of the no EE/ no cue group $(p<0.05)$; moreover, the EE/no cue and EE/ cue groups were significantly decreased compared to the no EE/cue group $(p<0.05)$. Therefore, the combination of $\mathrm{EE}$ and cue exhibited the lowest freezing time. EE or cue manipulations could suppress freezing behavior. The EE manipulation likely reduced much more freezing time when compared to the cue manipulation (Figure 5).

To analyze mean $( \pm$ SEM) total freezing time that was merged with the behavioral data overall sessions 1-3, twoway ANOVA was conducted to show that significant differences occurred in the factor of $\operatorname{EE}(F(1,20)=70.73, p<0.05$ ) , cue $(F(1,20)=14.14, p<0.05)$, and the interaction of EE and cue $(F(1,20)=3.25, p<0.05)$. Furthermore, one-way ANOVA indicated that the factor of group was significant differences $(F(3,20)=29.37, p<0.05)$. Post hoc Tukey's HSD tests showed that the no EE/cue, EE/no cue, and EE/ cue were significantly decreased in total freezing time compared to the no EE/no cue group $(p<0.05)$. The total freezing time of the EE/no cue and EE/cue group was significantly decreased than that of the no EE/cue group $(p<0.05)$. However, the freezing time of the EE/no cue group was not significantly different from that of the EE/ cue group $(p>0.05$; Figure 6$)$. The similar evidence as 


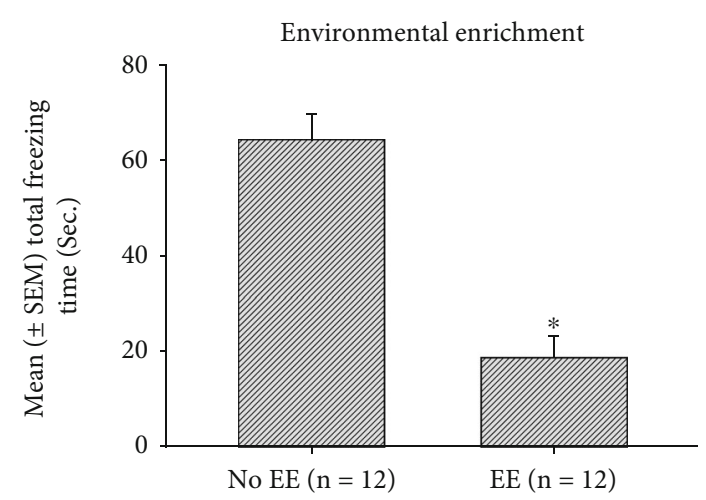

Figure 2: Assessments of the mean $( \pm$ SEM) total freezing time (Sec.) for the min effect of $\mathrm{EE}$ in the no $\mathrm{EE}(n=12)$ and $\mathrm{EE}$ $(n=12)$ conditions. EE: environmental enrichment. ${ }^{*} p<0.05$ indicates significant differences compared to the no EE condition.

mentioned above that the EE manipulation might be effective to reduce total freezing time. The combination of EE and cue treatments was likely the greatest reduction in total freezing behavior.

3.2. Immunohistochemical Staining with c-Fos Protein Expression. To examine the involvements of selected brain areas in the $\mathrm{EE}$, cue, or the interaction of $\mathrm{EE}$ and cue, a 2 $\times 2$ two-way ANOVA was conducted for the c-Fos expression after testing freezing behavior. The results of the EE manipulation showed that the c-Fos expression was significant increases in Cg1, IL, and NAc but decreases in BLA compared to the no EE manipulation $(p<0.05)$; the nonsignificant $c$-Fos expression occurred in the PrL, CA1, CA3, and DG $(p>0.05)$. The cue manipulations showed that the c-Fos expression was significant decreases in the Cg1, PrL, IL, CA1, CA3, and DG compared to the no cue manipulation $(p<0.05)$; nonsignificant differences occurred in the NAc and BLA $(p>0.05)$. The EE and cue interactions were significant differences in the C1g and NAc $(p<0.05)$; however, the other brain areas did not show significant differences $(p>0.05$; Table 2$)$.

On the other hand, one-way ANOVA was conducted to analyze the c-Fos expression in the selected brain areas. The results showed that a significant difference occurred in the factor of the group $(F(3,12)=29.35, p<0.05)$. In post hoc Tukey's HSD tests, the Cg1 showed that the EE/no cue was significantly increased in the c-Fos expression than the no $\mathrm{EE} /$ no cue and no EE/cue groups $(p<0.05)$; the no EE/cue and EE/cue were significantly decreased in the c-Fos expression compared to the no EE/no cue group $(p<0.05)$; the EE/ cue was significantly decreased in the c-Fos expression than the EE/cue group $(p<0.05$; Figure $7(a))$.

In the PrL c-Fos expression measurements, a significant difference occurred in the factor of group $(F(3,12)=5.21$, $p<0.05)$. In post hoc Tukey's HSD tests, the results showed that the EE/no cue was significantly increased in the c-Fos expression than the no EE/cue group $(p<0.05$; Figure $7(b))$.

In the IL c-Fos expression measurements, a significant difference occurred in the factor of group $(F(3,12)=4.44$, $p<0.05)$. In post hoc Tukey's HSD tests, the results showed

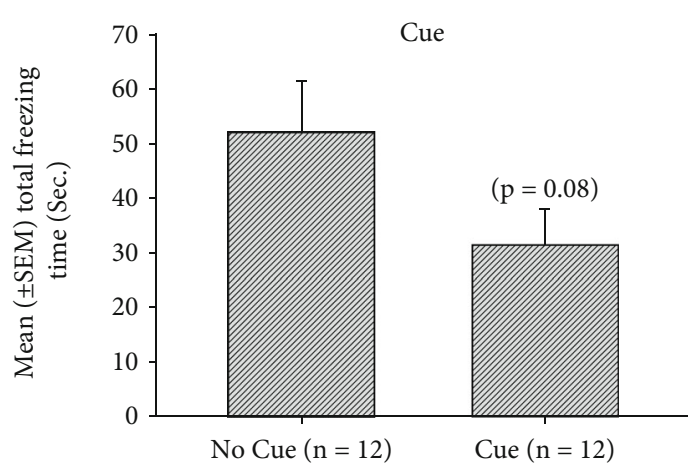

FIgUre 3: Assessments of mean ( \pm SEM) total freezing time (Sec.) for the main effect of the cue in the no cue $(n=12)$ and cue $(n=12)$ conditions. ${ }^{*} p<0.05$ indicates significant differences compared to the no cue condition.

that the EE/no cue was significantly increased in the c-Fos expression than the no EE/cue group $(p<0.05$; Figures 7(c) and 8).

In the NAc c-Fos expression measurements, a significant difference occurred in the factor of group $(F(3,12)=4.18$, $p<0.05)$. In post hoc Tukey's HSD tests, the results showed that the EE/no cue was significantly increased in the c-Fos expression than the no EE/no cue group $(p<0.05$; Figure 9(a)).

In the BLA c-Fos expression measurements, a significant difference occurred in the factor of the group $(F(3,12)=4.32, p<0.05)$. In post hoc Tukey's HSD tests, the results showed that the EE/cue was significantly decreased in the c-Fos expression than the No EE/no cue group ( $p<0.05$; Figures 9(b) and 10).

In the CA1 c-Fos expression assessments, a significant difference occurred in the factor of group $(F(3,12)=11.40$ $, p<0.05)$. In post hoc Tukey's HSD tests, the results showed that the no EE/cue and EE/cue groups were significantly decreased in the c-Fos expression than the no EE/no cue group $(p<0.05)$; the EE/no cue and EE/cue were significantly increased and decreased in the $c$-Fos expression, respectively, compared to the no EE/cue group $(p<0.05$; Figure 11(a)).

In the CA3 c-Fos expression assessments, a significant difference occurred in the factor of group $(F(3,12)=5.81$, $p<0.05)$. In post hoc Tukey's HSD tests, the results showed that the no EE/cue group was significantly decreased in the $c$-Fos expression than the no EE/no cue group $(p<0.05)$; the EE/no cue was significantly increased in the $c$-Fos expression compared to the no EE/cue group $(p<0.05$; Figure 11(b)).

In the DG c-Fos expression assessments, a significant difference occurred in the factor of group $(F(3,12)=6.89$, $p<0.05]$. In post hoc Tukey's HSD tests, the results showed that the EE/no cue group was significantly increased in the c-Fos expression than the no EE/cue group $(p<0.05)$; the $\mathrm{EE} / \mathrm{cue}$ was significantly decreased in the $\mathrm{c}$-Fos expression compared to the EE/no cue group ( $p<0.05$; Figures 11(c) and 12).

Therefore, the Cg1, IL, and NAc were upregulated in the c-Fos expression, but the BLA was a downregulation in the 


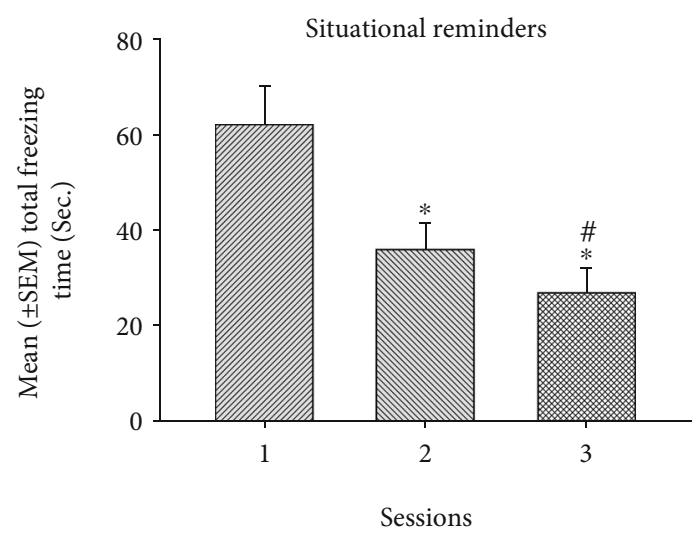

Figure 4: The mean ( \pm SEM) total freezing time (Sec.) in the situational reminders for sessions 1-3. ${ }^{*} p<0.05$ indicates significant differences compared to session 1. \#p<0.05 indicates significant differences compared to session 2 .

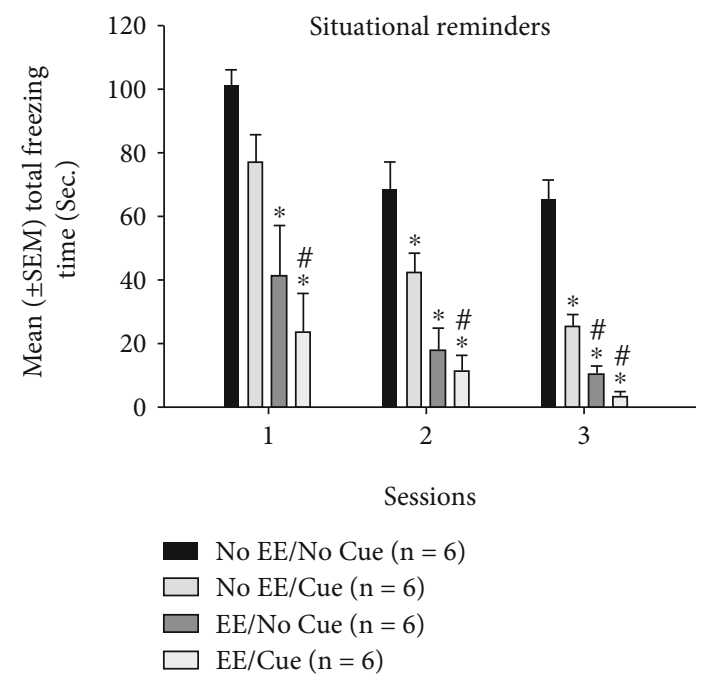

Figure 5: Mean ( \pm SEM) freezing time for the situational reminder in sessions 1-3 for the no EE/no cue, no EE/cue, EE/no cue, and EE/ cue groups ( $n=6$, per group). EE: environmental enrichment. ${ }^{*} p$ $<0.05$ indicates significant differences compared to the no EE/no cue group; $\# p<0.05$ indicates significant differences compared to the no EE/cue group.

c-Fos expression through the EE manipulations. The Cg1, PrL, IL, CA1, CA3, and DG were a downregulation in the c-Fos expression via the cue manipulations. Notably, the Cg1 and NAc were significant differences in the c-Fos expression under the interaction between $\mathrm{EE}$ and cue.

3.3. $q R T-P C R$ Assessment for the c-Fos $m R N A$ Expression. To test the c-Fos mRNA expression of EE, cue, and EExcue in the mPFC, NAc, amygdala, and hippocampus, a $2 \times 2$ twoway ANOVA was conducted after testing freezing behavior. The results showed that EE manipulations were significant increases in the mPFC $(F(1,12)=4.68, p<0.05)$ and NAc $(F(1,12)=14.01, p<0.05)$; however, there was significant decreases in the amygdala $(F(1,12)=10.98, p<0.05)$ compared to the no EE manipulations. There were nonsignifi-

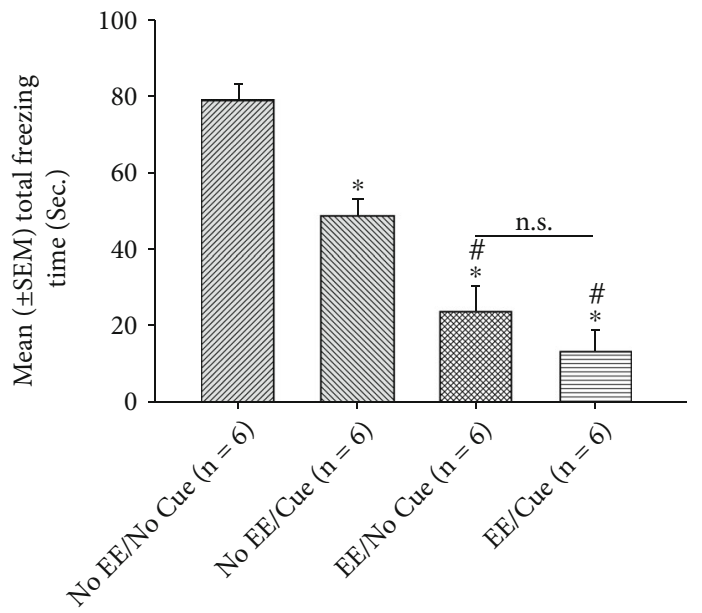

Figure 6: Mean ( \pm SEM) total freezing time from the merged data in sessions 1-3 for the no EE/no cue, no EE/cue, EE/no cue, and EE/ cue groups ( $n=6$, per group). EE: environmental enrichment. ${ }^{*} p$ $<0.05$ indicates significant differences compared to the no EE/no cue group; $\# p<0.05$ indicates significant differences compared to the no EE/cue group. (n.s.): nonsignificant differences.

cant differences between the EE and no EE manipulations in the hippocampus $(F(1,12)=0.26, p>0.05)$. The cue manipulation was significant decreases in the $\mathrm{mPFC}$ $(F(1,12)=14.01, \quad p<0.05)$ and the hippocampus $(F(1,12)=16.76, p<0.05)$ compared to the no EE manipulation. The cue showed nonsignificant differences in the NAc $(F(1,12)=2.52, \quad p>0.05) \quad$ and the amygdala $(F(1,12)=1.59, p>0.05)$. The interaction of EE and cure reveled the nonsignificant differences in the $\mathrm{mPFC}$ $(F(1,12)=1.95, p>0.05)$, the NAc $(F(1,12)=1.82, p>$ $0.05)$, the amygdala $(F(1,12)=1.89, p>0.05)$, and the hippocampus $(F(1,12)=0.27, p>0.05$; Table 3$)$.

Furthermore, one-way ANOVA analysis was conducted to analyze the c-Fos mRNA expression in the mPFC, NAc, amygdala, and hippocampus among the no EE/no cue, no $\mathrm{EE} /$ cue, EE/no cue, and EE/cue groups. For the $\mathrm{mPFC}$, the factor of group was significant differences $(F(3,12)=6.88$, $p<0.05)$. The post hoc Tukey tests indicated that the c-Fos mRNA expression of the EE/no cue group was significant increases than that of the no EE/no cue group $(p<0.05)$; moreover, the c-Fos mRNA expression of the EE/cue group was significantly decreased compared to the EE/no cue group $(p<0.05$; Figure 13(a)). The result indicated that the EE manipulation is likely to enhance the c-Fos mRNA expression; however, the cue conduction might decrease the $\mathrm{c}$-Fos mRNA expression in the mPFC.

To test the c-Fos mRNA expression of the NAc, one-way ANOVA analysis showed that significant differences occurred in the factor of the group $(F(3,12)=5.85, p<$ $0.05)$. The post hoc Tukey test showed that the c-Fos mRNA expression of the EE/no cue group was significantly increased compared to that of the no EE/no cue group $(p<0.05$; Figure 13(b)), indicating that EE manipulations increased the c-Fos mRNA expression in the NAc.

One-way ANOVA was performed to test the amygdala's c-Fos mRNA expression in the EE and cue manipulations. 
TABLE 2: Effects of environmental enrichment and cue for PTSD in the c-Fos expression for selected brain areas in the situational reminder phase.

\begin{tabular}{lcccccccc}
\hline & Cg1 & PrL & IL & NAc & BLA & CA1 & CA3 & DG \\
\hline Environmental enrichment & $\uparrow$ & - & $\uparrow$ & $\uparrow$ & $\downarrow$ & - & - & - \\
Cue & $\downarrow$ & $\downarrow$ & $\downarrow$ & - & - & $\downarrow$ & $\downarrow$ & $\downarrow$ \\
Interaction of Cue and environmental enrichment & + & - & - & + & - & - & - & - \\
\hline
\end{tabular}

Note: $(\uparrow)$ : increases; $(\downarrow)$ : decreases; (-): nonsignificant difference; $(+): p<0.05$.

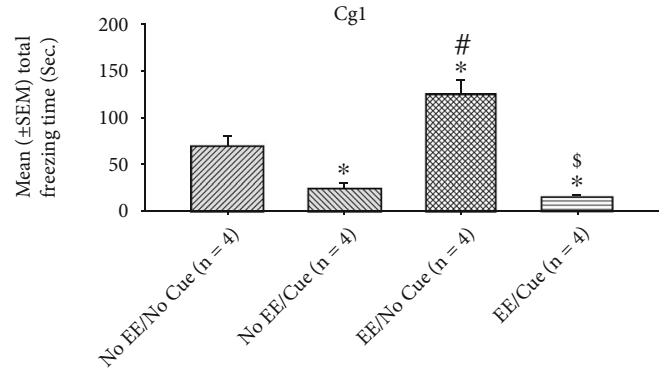

(a)

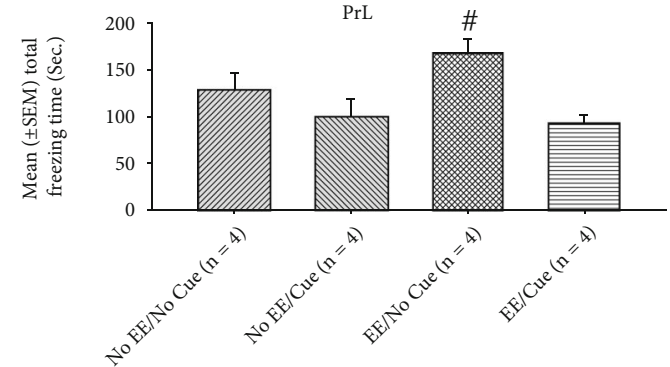

(b)

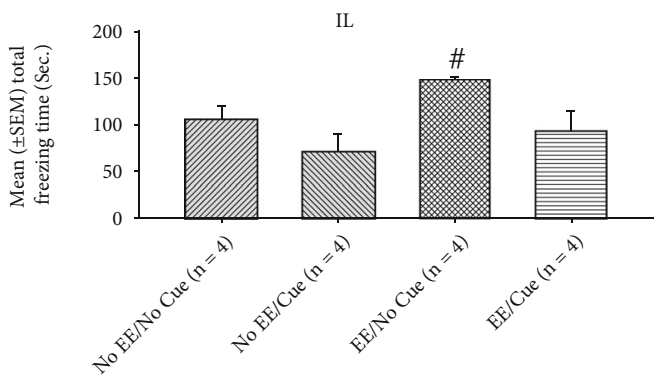

(c)

FIgure 7: Mean ( \pm SEM) c-Fos expression in the Cg1 (a), PrL (b), and IL (c) for the no EE/no cue $(n=6)$, no EE/cue $(n=6)$, EE/no cue $(n=6)$, and EE/cue groups $(n=6)$. EE: environmental enrichment. ${ }^{*} p<0.05$ indicates significant differences compared to the no EE/no cue group; $\# p<0.05$ indicates significant differences compared to the no EE/cue group.

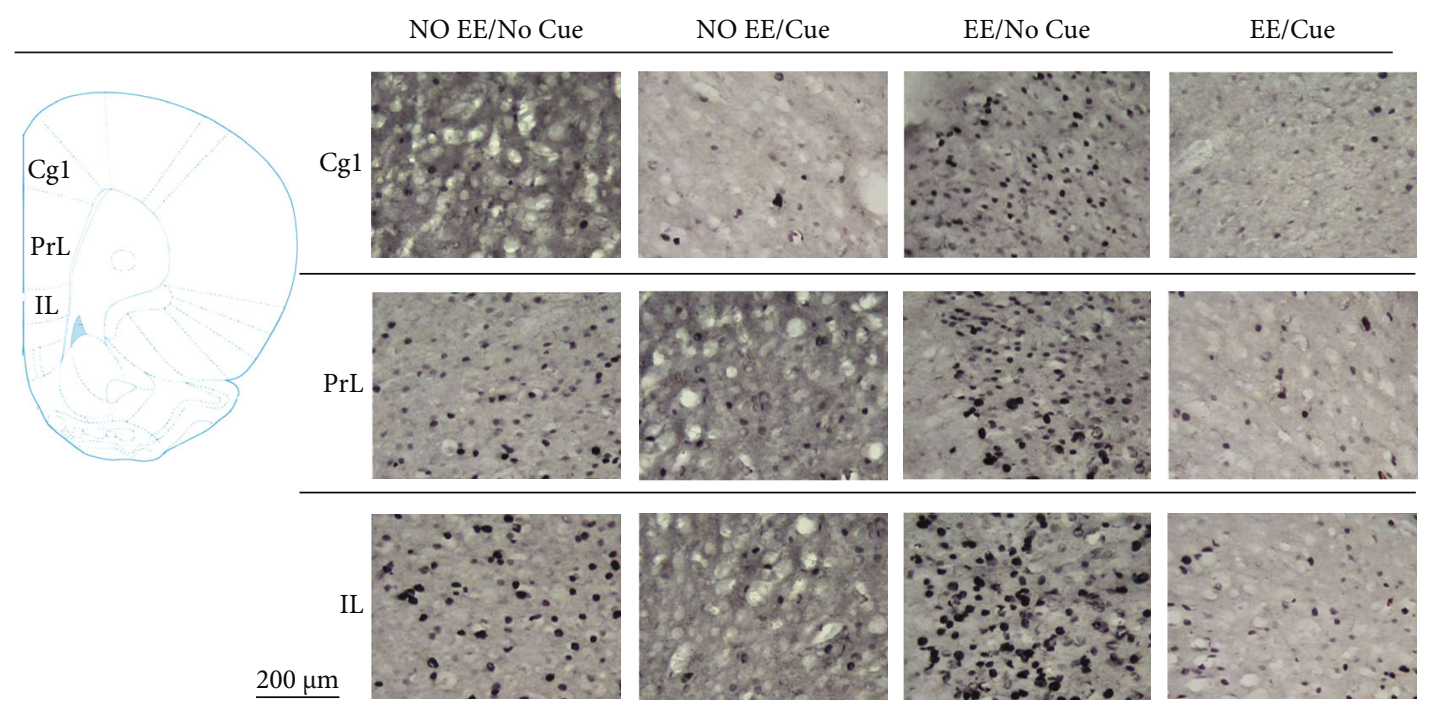

Figure 8: (a) A schematic brain atlas for the Cg1, PrL, and IL of the mPFC. (b) Representative photomicrographs of the c-Fos expression for the Cg1, PrL, and IL in the no EE/no cue, no EE/cue, EE/no cue, and EE/cue groups ( $n=4$, per group). Scale bar represents $200 \mu \mathrm{m}$. 


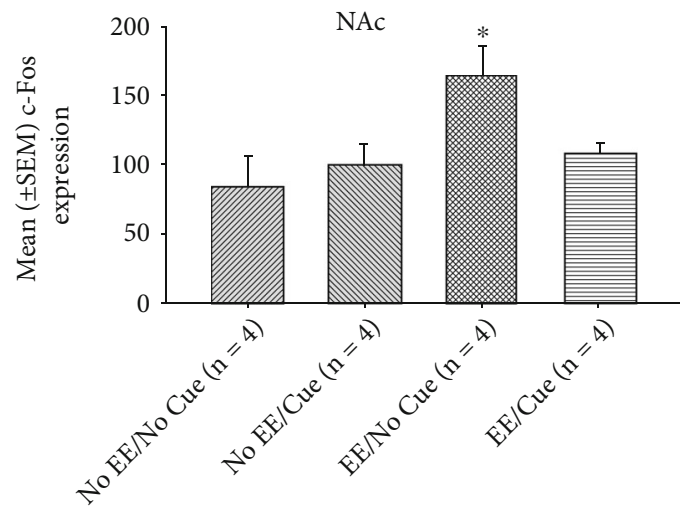

(a)

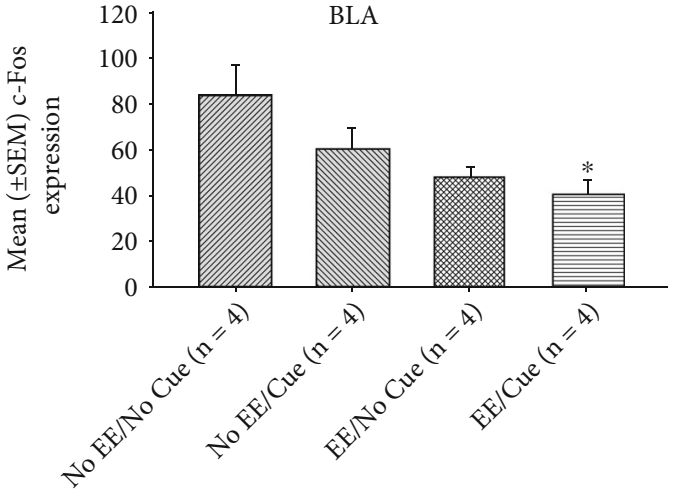

(b)

Figure 9: Mean ( \pm SEM) c-Fos expression in the NAc (a) and BLA (b) for the no EE/no cue $(n=6)$, no EE/cue $(n=6)$, EE/no cue $(n=6)$, and EE/cue groups $(n=6)$. EE: environmental enrichment. ${ }^{*} p<0.05$ indicates significant differences compared to the no EE/no cue group; $\# p<0.05$ indicates significant differences compared to the no EE/cue group.

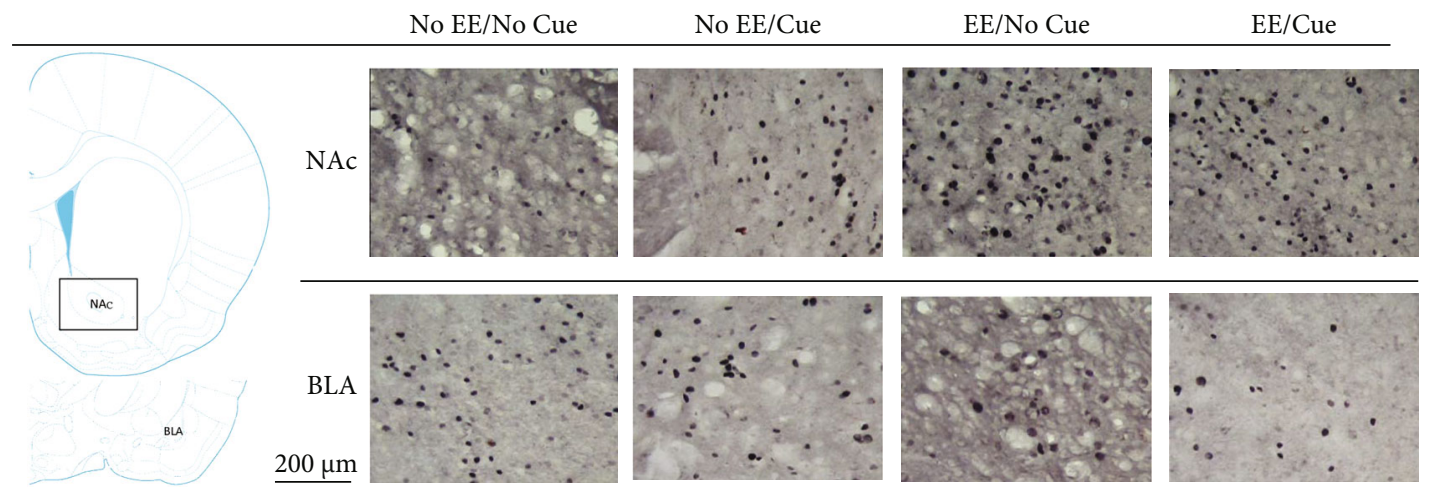

Figure 10: (a) A schematic brain atlas for the NAc and the amygdala's BLA. (b) Representative photomicrographs of the c-Fos expression for the NAc and the amygdala's BLA in the no EE/no cue, no EE/cue, EE/no cue, and EE/cue groups ( $n=4$, per group). Scale bar represents $200 \mu \mathrm{m}$.

The results showed that the factor of group had significant differences $(F(3,12)=4.82, p<0.05)$. The post hoc Tukey tests indicated significant decreases in the $\mathrm{EE} / \mathrm{no}$ cue and the $\mathrm{EE} /$ cue groups compared to the no $\mathrm{EE} /$ no cue group $(p<0.05$; Figure 13(c)), indicating that the EE manipulation decreased the c-Fos mRNA expression in the amygdala.

To test c-Fos mRNA of the hippocampus in the EE and cue manipulations, one-way ANOVA analysis was conducted to show that only the $\mathrm{EE} /$ cue group was significant decreases in the c-Fos mRNA expression compared to the no EE/no cue and the EE/no cue groups $(p<0.05)$, respectively (Figure 13(d)). Under cue manipulation, the c-Fos mRNA expression of the hippocampus was significantly decreased.

In conclusion, the results of the c-Fos mRNA expression were similar to the findings of the c-Fos protein expression in the mPFC, NAc, amygdala, and the hippocampus (see Tables 2 and 3).

\section{Discussion}

The EE manipulations can reduce footshock-induced fear behavior, and the cue manipulation also reduces fear behav- ior in PTSD symptoms. The present data showed that the EE is better than the cue manipulation to ameliorate fear behavior in PTSD. The combination of EE and cue induced the most significant reduction effect in fear behavior. Cue manipulation is likely a novel treatment to reduce PTSD symptoms, in particular, to fear behavior.

The c-Fos expression data showed that the Cg1, IL, and NAc upregulated the c-Fos expression in fear behavior; however, the BLA downregulated the c-Fos expression in fear behavior for PTSD symptoms through the EE manipulations, indicating the Cg1, IL, NAc, and BLA controlled the EE-induced fear reduction. In contrast, the Cg1, PrL, IL, CA1, CA3, and DG were downregulated in the c-Fos expression after the cue manipulations in fear behavior, indicating the Cg1, PrL, IL, CA1, CA3, and DG contributed to a cueinduced fear reduction in PTSD. The EE and cue had significant interactions in the $\mathrm{Cg} 1$ and NAc for the c-Fos expression. Accordingly, the Cg1 and NAc were involved in the interaction of $\mathrm{EE}$ and cue.

The data of the c-Fos mRNA expression were similar to the findings of the c-Fos protein expression. EE manipulations upregulated the c-Fos mRNA expression in the mPFC and the NAc; however, it downregulated the c-Fos mRNA 


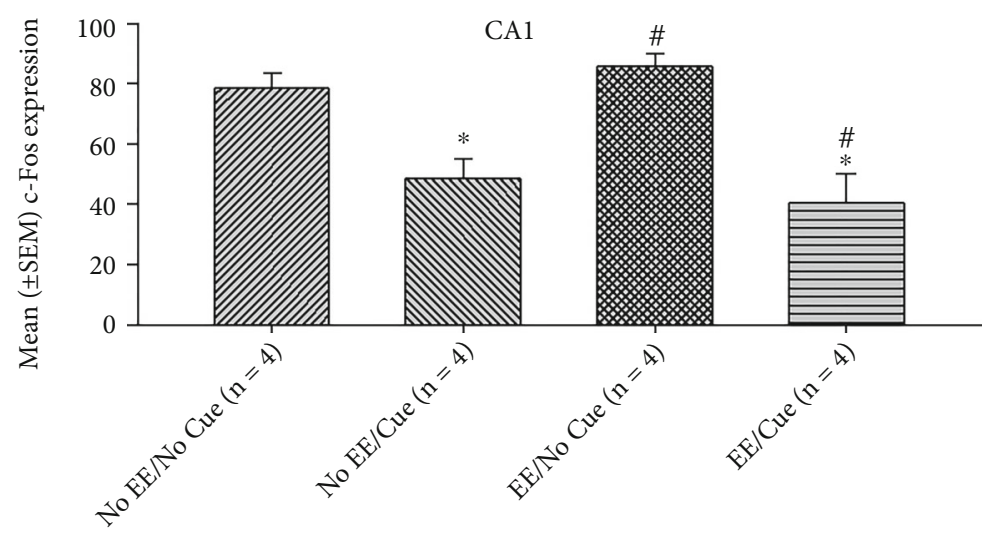

(a)

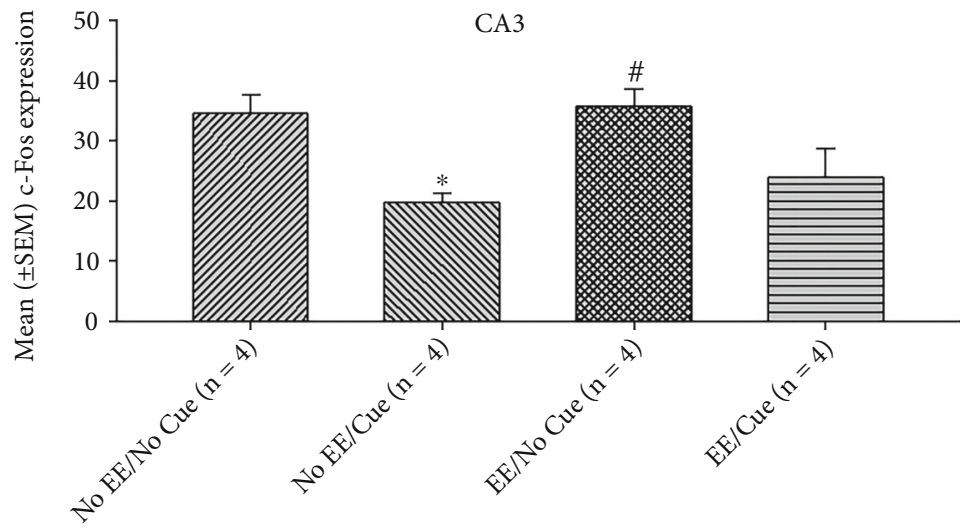

(b)

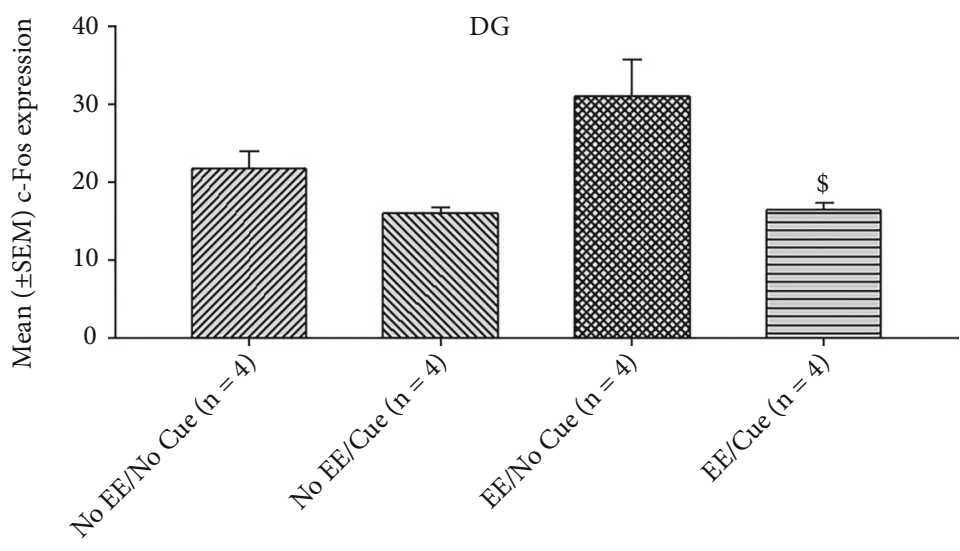

(c)

Figure 11: Mean ( \pm SEM) c-Fos expression in the CA1 (a), CA3 (b), and DG (B) for the no EE/no cue $(n=6)$, no EE/cue $(n=6)$, EE/no cue $(n=6)$, and EE/cue groups $(n=6)$. EE: environmental enrichment. ${ }^{*} p<0.05$ indicates significant differences compared to the no EE/no cue group; $\# p<0.05$ indicates significant differences compared to the no EE/cue group.

expression in the amygdala. Cue manipulations downregulated the c-Fos mRNA expression in the MPFC and hippocampus. EE and cue interactions did not affect c-Fos mRNA expression in the mPFC, NAc, amygdala, and hippocampus.

4.1. Environmental Enrichment, Cue, and Combination of Environmental Enrichment and Cue to Ameliorate Footshock-Induced Fear Behavior in PTSD Symptoms. In the present data, the EE manipulations ameliorated footshock-induced fear behavior. Therefore, the present data of EE is consistent with the previous findings [33]. Dependent on the previous data, a growing body of evidence showed that the EE manipulations decreased fear- and stress-related behaviors in different levels [6-11, 34]. For example, the behavioral test of EE showed that long-term EE suppressed neonatal isolation-induced contextual freezing, but this type of EE did not change single prolonged 


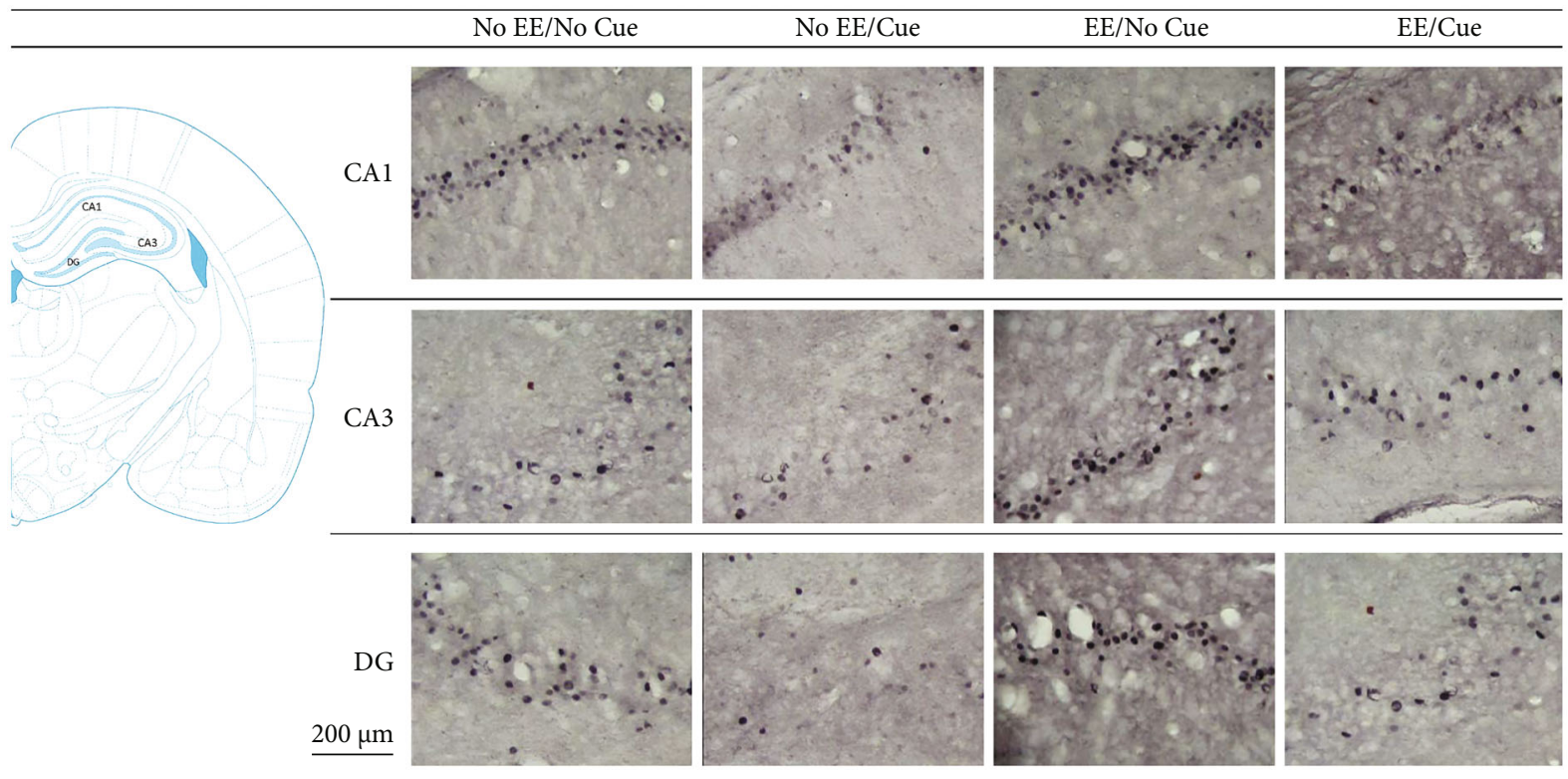

Figure 12: (a) A schematic brain atlas for the Cg1, CA3, and DG of the hippocampus. (b) Representative photomicrographs of the c-Fos expression for the CA1, CA3, and DG of the hippocampus in the no EE/no cue, no EE/cue, EE/no cue, and EE/cue groups $(n=4$, per group). Scale bar represents $200 \mu \mathrm{m}$.

TABLE 3: Effects of environmental enrichment and cue for PTSD in the normalized c-Fos mRNA expression for selected brain areas in the situational reminder phase.

\begin{tabular}{|c|c|c|c|c|}
\hline & $\mathrm{mPFC}$ & NAc & Amygdala & Hippocampus \\
\hline Environmental enrichment & $\uparrow$ & $\uparrow$ & $\downarrow$ & - \\
\hline Cue & $\downarrow$ & - & - & $\downarrow$ \\
\hline Interaction of Cue and environmental enrichment & - & - & - & - \\
\hline
\end{tabular}

Note: $(\uparrow)$ : increases; $(\downarrow)$ : decreases; (-): nonsignificant difference; $(+)$ : $p<0.05$.

stress related to anxiety behavior or analgesia. Moreover, EE exposures showed to reduce deep-brain stimulation-induced resistant depression and PTSD in the animal model [6]. Alternatively, the examinations of the brain system in EE ameliorations showed that EE could facilitate the negative feedback regulation of the HPA axis for stress rats, and it ameliorated the abnormal behaviors via downregulation of glucocorticoid receptor expression in the hippocampus and hypothalamus [7]. In another study, activations of serotonin and neuropeptide systems through EE manipulations were shown to reduce anxiety behaviors in the PTSD animal model [11]. Regarding the examination of EE manipulations in neuronal levels, animals with EE exposures increased hippocampal cell proliferation and recovered normal behaviors in PTSD symptoms [34]. Additionally, short-term EE exposures potentiated the extinction process of fear via regulating neuropeptide Y-Y1 receptors in the hippocampus [10]. A previous study has demonstrated that EE exposures ameliorated the extended volumes of the hippocampus and central amygdala caused by traumatic stress [8]. Therefore, the EE manipulations may be effectively nonpharmacological interventions in reducing footshock-induced stress or anxiety in behavior, brain systems, and neuronal levels.

On the other hand, manipulating the cue stimulus is likely a novel approach to examine the amelioration of footshock-induced fear behavior. The present results showed that the cue exposure showed a significant decrease in fear behavior induced by footshock; however, this suppressed effect by the cue stimulus was not higher than that of the EE manipulation. The single cue stimulus may cause the different effects between $\mathrm{EE}$ and cue, but the EE is composed of a variety of stimuli, including sensory, physical, social, and cognitive components $[12,13]$. Therefore, the EE manipulation is similar to the real contextual stimulus.

\subsection{Cue and Environmental Enrichment: An Occasion Setter} or a CS Associated with the US? These inconsistent data for the EE and cue manipulations should be explained by that the novelty and salient property of stimulus from the EE exposures might be the crucial factor to ameliorate footshock-induced fear behavior $[35,36]$. However, the cue stimulus is short of comprehensive and complete stimuli; instead, the cue exposure only has a single stimulus dimension, causing a weaker novelty and salient property to reduce footshock-induced fear behavior. Interestingly, the combination of $\mathrm{EE}$ and cue revealed the highest reduction in footshock-induced fear behavior. What reason is the combination of $\mathrm{EE}$ and cue due to the highest reduction for footshock-induced fear behavior? It might be two possibilities. One possible reason is that the cue stimulus is served 


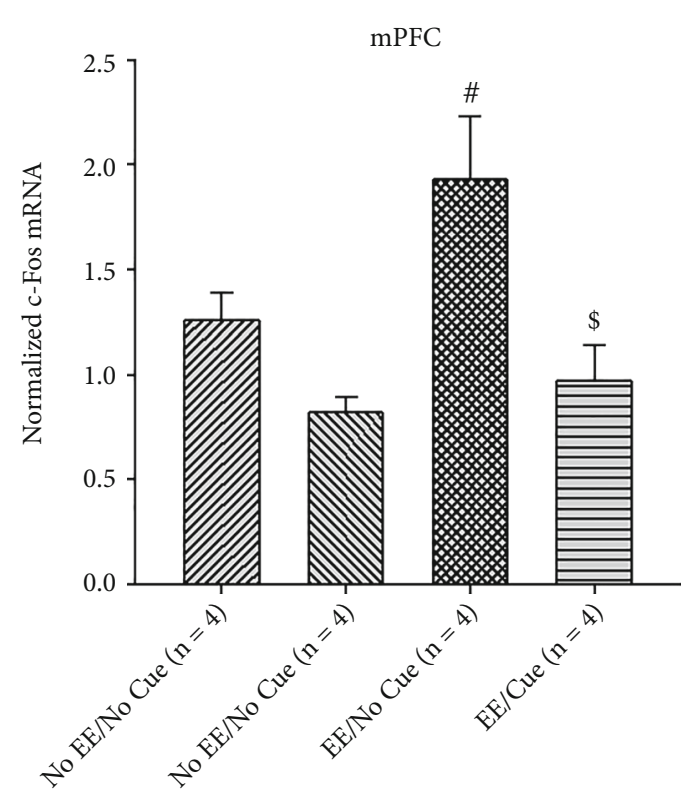

(a)

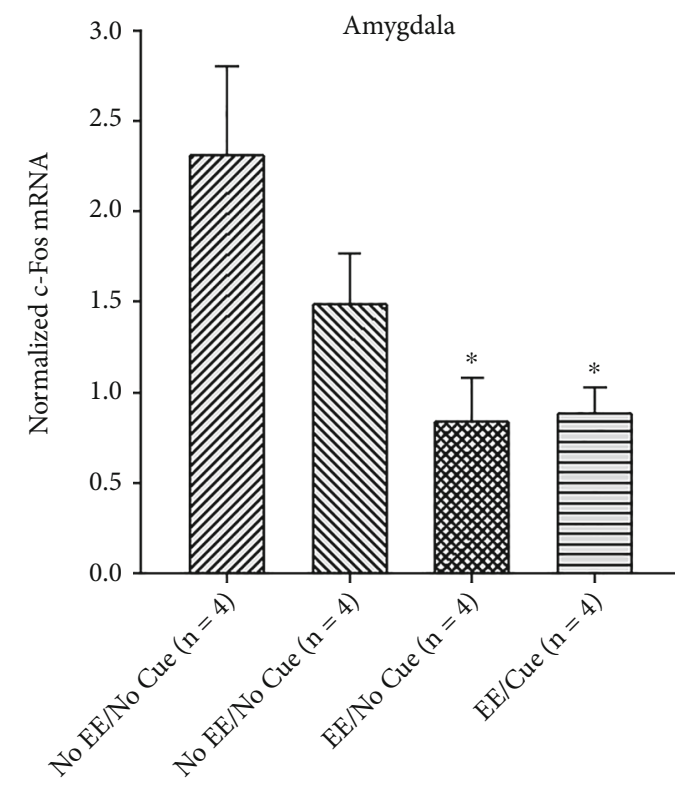

(c)

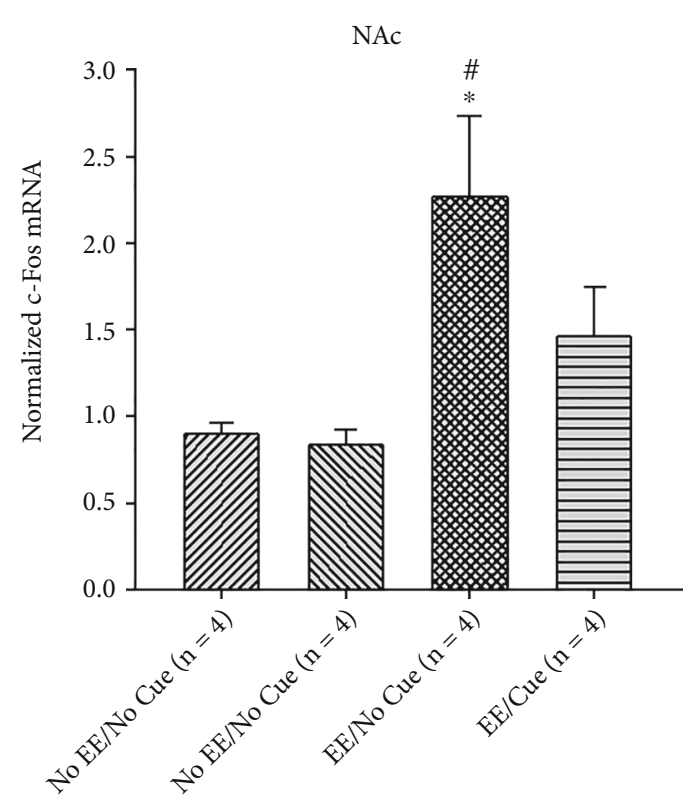

(b)

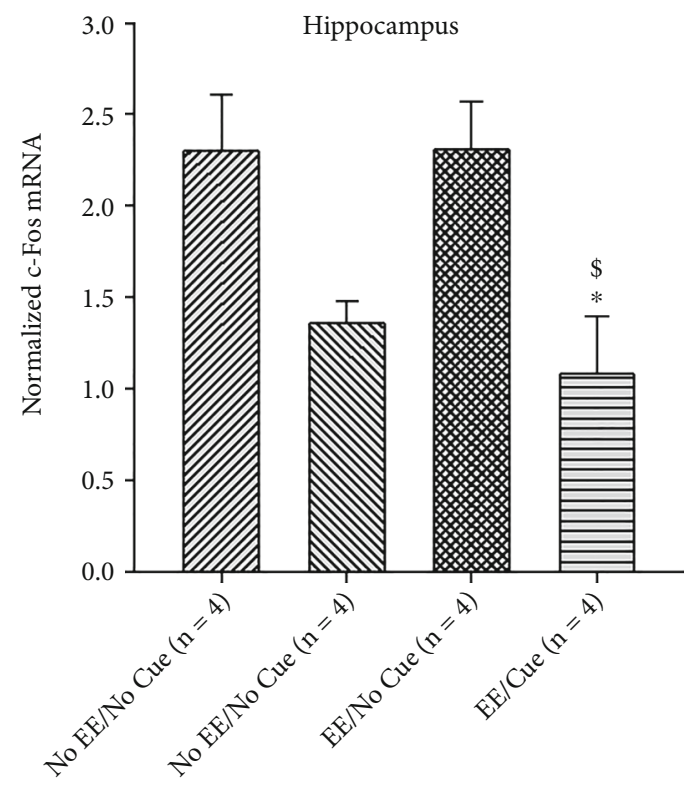

(d)

Figure 13: Mean ( \pm SEM) normalized c-Fos mRNA in the mPFC (a), NAc (b), amygdala (c), and hippocampus (d) for the no EE/no cue $(n=4)$, no EE/cue $(n=4), \mathrm{EE} /$ no cue $(n=4)$, and EE/cue groups $(n=4)$. EE: environmental enrichment. ${ }^{*} p<0.05$ indicates significant differences compared to the no EE/no cue group; $\# p<0.05$ indicates significant differences compared to the no EE/cue group. $\$ p<0.05$ indicates significant differences compared to the $\mathrm{EE} /$ no cue group.

as a conditioned stimulus (CS) to interact with the unconditioned stimulus (US) to modulate the strengths of the conditioned response (CR) by the rule of classical conditioning [37]. Another possible reason is that the cue stimulus is an occasion setter to enhance the CR, fear behavior. Based on the occasion setting theory, the cue stimulus itself was not involved in the association with the US; however, the occasion setter stimulus only modulates the strength of the CR $[38,39]$. According to the present study, the cue stimulus seemingly contributed to the association with the US. There- fore, whether the cue stimulus is an occasion setter or a CS remains scrutinized in further studies.

4.3. Involvements of the $m P F C$, Amygdala, Hippocampus, and NAc in Environmental Enrichment, Cue, or the Combination of Environmental Enrichment and Cue in PTSD Fear Symptoms. To date, no research comprehensively examined how the mPFC, amygdala, and hippocampus contributed to suppressing EE or cue in PTSD symptoms and behaviors. However, amount of research has demonstrated 
that the mPFC's neuronal activity inhibited the amygdala's negative emotional responses, and the activation of the amygdala conveyed negative feedback information with the negative value to transmit into the mPFC, which explained the valences of this information [40]. This neural pathway of the mPFC projecting to the amygdala has been shown to regulate PTSD symptoms [41, 42]; moreover, the present data showed that the mPFC-amygdala pathway was seemingly involved in the suppression of EE or cue to PTSD symptoms. On the other hand, the hippocampus was suggested to modulate spatial learning and configural memory $[19,43]$, and the hippocampus is essential to process memory contents in the process of consolidation from the short-term memory to the long-term memory $[44,45]$. Accordingly, the hippocampus was acquired by the amygdala's information to modulate the formation of the configural memory and spatial learning. This result is likely consistent with the recent findings that the amygdala interacted with the hippocampus to mediate emotional learning and memory and PTSD symptoms [46]. The amygdala's pathway with the hippocampus might be another crucial neural pathway for PTSD symptoms.

In the reduced effect of the EE in PTSD fear behavior, it was shown that the Cg1 and IL (but not the PrL) of the mPFC were increased in the c-Fos expression, and it indicated that after chronic EE exposures, the mPFC's executive and inhibitory functions interfered with the activity of the amygdala's BLA, which presented decreases in the c-Fos expression. Decreased c-Fos expression of the BLA indicated that the BLA inhibited the negative emotional responses following the EE exposures.

Alternatively, the present data showed that the c-Fos expression of the NAc significantly increased, indicating that EE exposures might induce dopamine neurotransmitters secretions in the NAc due to the novelty or saliency effects following EE exposures [36, 47, 48]. This novelty and saliency of EE's property may be the critical point that the EE manipulations could reduce the symptoms of psychiatric disorders (e.g., PTSD and depression) or neurological diseases (Parkinson's disease or Alzheimer's disease) [35]. How did the novelty and saliency of EE affect the symptoms of psychiatric and neurological diseases which remain to be investigated further?

In particular, the cue stimulus manipulations showed different results that the $\mathrm{Cg} 1$, PrL, and IL of the mPFC and the CA1, CA3, and DG of the hippocampus decreased the c-Fos expression compared to the EE manipulations. Accordingly, the cue manipulations may play a different role in the reduction of footshock-induced fear behavior when compared to the EE manipulations. The cue stimulus is not contextual, and it is likely an occasion setting to modulate fear behavior induced by footshock in the PTSD animal model $[39,49]$. Thus, the occasion setter role of the cue fully decreased the c-Fos expression in the whole subregions of the mPFC and the hippocampus. The results indicated that the cue stimulus interfered with the executive and inhibitory functions resulted in the mPFC dysfunctions; moreover, the cue stimulus decreased the c-Fos expression in the CA1, CA3, and DG of the hip- pocampus, indicating the hippocampus revealed dysfunctions in spatial learning and configural memory. Accordingly, the c-Fos data of the cue stimulus manipulations revealed a worse reduction to footshock-induced fear behavior than those of the EE manipulation and the combination of the $\mathrm{EE}$ and cue manipulations.

In the examinations of the interaction between $\mathrm{EE}$ and cue, the results showed that significant differences occurred in the Cg1 of the mPFC and the NAc to reduce footshockinduced fear behavior. Why did the c-Fos expression of the $\mathrm{Cg} 1$ and NAc produce an interaction between the $\mathrm{EE}$ and cue manipulations? It is due to the property of the Cg1 governing the stress or anxiety behaviors and the property of the NAc involving the reward or reinforcement process. This emerged issue should be concerned with further studies.

\section{Conclusion}

The EE and cue suppressed footshock-induced fear behavior; however, the EE manipulation produced a more potent suppression in footshock-induced fear behavior compared to the cue manipulation; although, the cue stimulus also reduced footshock-induced fear behavior. These data supported the previous findings $[6,8,11,35,36]$. For example, EE exposures reduced traumatic stress and increased the volume of the hippocampus and central amygdala [8]; moreover, it ameliorated anxiety [11], depression [6], PTSD symptoms [6], and footshock-induced fear behaviors [35, 36 in the animal model. Notably, the combination of EE and cue manipulations produced the strongest reduction in footshock-induced fear behavior. Under the EE manipulations, c-Fos upregulation occurred in the Cg1, IL, and NAc, but c-Fos downregulation occurred in the BLA. Under cue manipulations, c-Fos downregulation occurred in the Cg1, PrL, IL, CA1, CA3, and DG. The data of the c-Fos mRNA expression were similar with the data of the c-Fos protein expression. The c-Fos mRNA expression was upregulation in the mPFC and amygdala in the EE manipulations; the amygdala was downregulated for the c-Fos mRNA expression in the EE manipulations. Cue manipulations were decreases in the c-Fos mRNA expression for the mPFC and hippocampus. The present findings are likely to offer contributions for novel and nonpharmacological treatments to PTSD symptoms. The present data might help understand the amelioration mechanisms of PTSD fear symptoms in the brain.

\section{Data Availability}

The raw data can be accessed through the following link: https://www.dropbox.com/sh/r11cj1scrvggykz/AAC_ MvtjwTIMAAQYb9p92p0a?dl=0.

\section{Conflicts of Interest}

The authors declare that they have no competing interests. 


\section{Authors' Contributions}

YHY contributed to the conceptualization, methodology, validation, investigation, data curation, and project administration. YSL contributed to the project administration. CYO contributed to the project administration. KCC contributed to the data curation. FCC contributed to the project administration. ACT contributed to the statistic analysis supports. ACWH contributed to the conceptualization, methodology, formal analysis, investigation, writing-review and editing, and funding acquisition.

\section{Acknowledgments}

The present study was supported by funding granted from the Ministry of Science and Technology, Taiwan to Dr. Andrew Chih Wei Huang (MOST 110-2410-H-431-004) and Dr. Arthur C. Tasi (MOST 107-2118-M-001-006-MY2).

\section{References}

[1] H. Cohen, N. Kozlovsky, C. Alona, M. A. Matar, and Z. Joseph, "Animal model for PTSD: from clinical concept to translational research," Neuropharmacology, vol. 62, pp. 715-724, 2012.

[2] C. Grillon, S. M. Southwick, and D. S. Charney, "The psychobiological basis of posttraumatic stress disorder," Molecular Psychiatry, vol. 1, pp. 278-297, 1996.

[3] D. Kozaric-Kovacic, "Psychopharmacotherapy of posttraumatic stress disorder," Croatian Medical Journal, vol. 49, pp. 459-475, 2008.

[4] A. Siegmund and C. T. Wotjak, "Hyperarousal does not depend on trauma-related contextual memory in an animal model of posttraumatic stress disorder," Physiology \& Behavior, vol. 90, pp. 103-107, 2007.

[5] E. I. Flandreau and M. Toth, "Animal models of PTSD: a critical review," Current Topics in Behavioral Neurosciences, vol. 38, pp. 47-68, 2018.

[6] Y. Bhaskar, L. W. Lim, and R. Mitra, "Enriched environment facilitates anxiolytic efficacy driven by deep-brain stimulation of medial prefrontal cortex," Frontiers in Behavioral Neuroscience, vol. 12, p. 204, 2018.

[7] Z. Fan, J. Chen, L. Li et al., "Environmental enrichment modulates HPA axis reprogramming in adult male rats exposed to early adolescent stress," Neuroscience Research, vol. 172, pp. 63-72, 2021.

[8] Y. Golub, S. F. Kaltwasser, C. P. Mauch et al., "Reduced hippocampus volume in the mouse model of posttraumatic stress disorder," Journal of Psychiatric Research, vol. 45, pp. 650659, 2011.

[9] A. Imanaka, S. Morinobu, S. Toki, and S. Yamawaki, "Importance of early environment in the development of posttraumatic stress disorder-like behaviors," Behavioural Brain Research, vol. 173, pp. 129-137, 2006.

[10] G. Lach, M. A. Bicca, A. A. Hoeller, E. C. Santos, A. P. Costa, and T. C. de Lima, "Short-term enriched environment exposure facilitates fear extinction in adult rats: the NPY-Y1 receptor modulation," Neuropeptides, vol. 55, pp. 73-78, 2016.

[11] D. Ragu Varman and K. E. Rajan, "Environmental enrichment reduces anxiety by differentially activating serotonergic and neuropeptide Y (NPY)-ergic system in Indian field mouse
(Mus booduga): an animal model of post-traumatic stress disorder," PLoS One, vol. 10, article e0127945, 2015.

[12] J. C. Brenes, M. Lackinger, G. U. Hoglinger, G. Schratt, R. K. Schwarting, and M. Wohr, "Differential effects of social and physical environmental enrichment on brain plasticity, cognition, and ultrasonic communication in rats," The Journal of Comparative Neurology, vol. 524, no. 8, pp. 1586-1607, 2016.

[13] J. Nithianantharajah and A. J. Hannan, "Enriched environments, experience-dependent plasticity and disorders of the nervous system," Nature Reviews. Neuroscience, vol. 7, pp. 697-709, 2006.

[14] P. Sampedro-Piquero and A. Begega, "Environmental enrichment as a positive behavioral intervention across the lifespan," Current Neuropharmacology, vol. 15, pp. 459-470, 2017.

[15] D. T. Acheson, J. E. Gresack, and V. B. Risbrough, "Hippocampal dysfunction effects on context memory: possible etiology for posttraumatic stress disorder," Neuropharmacology, vol. 62, pp. 674-685, 2012.

[16] N. Kaouane, E. G. Ducourneau, A. Marighetto, M. Segal, and A. Desmedt, "False opposing fear memories are produced as a function of the hippocampal sector where glucocorticoid receptors are activated," Frontiers in Behavioral Neuroscience, vol. 14, p. 144, 2020.

[17] F. Steiger, F. Nees, M. Wicking, S. Lang, and H. Flor, "Behavioral and central correlates of contextual fear learning and contextual modulation of cued fear in posttraumatic stress disorder," International Journal of Psychophysiology, vol. 98, pp. 584-593, 2015.

[18] N. C. Huff and J. W. Rudy, "The amygdala modulates hippocampus-dependent context memory formation and stores cue-shock associations," Behavioral Neuroscience, vol. 118, pp. 53-62, 2004.

[19] L. E. Jarrard, "On the role of the hippocampus in learning and memory in the rat," Behavioral and Neural Biology, vol. 60, pp. 9-26, 1993.

[20] S. Tanimoto, T. Nakagawa, Y. Yamauchi, M. Minami, and M. Satoh, "Differential contributions of the basolateral and central nuclei of the amygdala in the negative affective component of chemical somatic and visceral pains in rats," The European Journal of Neuroscience, vol. 18, pp. 2343-2350, 2003.

[21] J. D. Bremner, "Traumatic stress: effects on the brain," Dialogues in Clinical Neuroscience, vol. 8, no. 4, pp. 445-461, 2006.

[22] S. H. Chang, Y. H. Yu, A. He, C. Y. Ou, B. C. Shyu, and A. C. W. Huang, "BDNF protein and BDNF mRNA expression of the medial prefrontal cortex, amygdala, and hippocampus during situational reminder in the PTSD animal model," Behavioural Neurology, vol. 2021, Article ID 6657716, 13 pages, 2021.

[23] B. M. Elzinga and J. D. Bremner, "Are the neural substrates of memory the final common pathway in posttraumatic stress disorder (PTSD)?," Journal of Affective Disorders, vol. 70, pp. 1-17, 2002.

[24] L. M. Shin, S. L. Rauch, and R. K. Pitman, "Amygdala, medial prefrontal cortex, and hippocampal function in PTSD," Annals of the New York Academy of Sciences, vol. 1071, pp. 67-79, 2006.

[25] M. M. Miller and B. S. McEwen, "Establishing an agenda for translational research on PTSD," Annals of the New York Academy of Sciences, vol. 1071, no. 1, pp. 294-312, 2006.

[26] N. Korem, R. Lange, C. J. Hillard, and I. Akirav, "Role of betacatenin and endocannabinoids in the nucleus accumbens in 
extinction in rats exposed to shock and reminders," Neuroscience, vol. 357, pp. 285-294, 2017.

[27] C. Piao, X. Deng, X. Wang, Y. Yuan, Z. Liu, and J. Liang, "Altered function in medial prefrontal cortex and nucleus accumbens links to stress-induced behavioral inflexibility," Behavioural Brain Research, vol. 317, pp. 16-26, 2017.

[28] J. C. Kung, T. C. Chen, B. C. Shyu, S. Hsiao, and A. C. Huang, "Anxiety- and depressive-like responses and c-fos activity in preproenkephalin knockout mice: oversensitivity hypothesis of enkephalin deficit-induced posttraumatic stress disorder," Journal of Biomedical Science, vol. 17, no. 1, p. 29, 2010.

[29] Y. H. Yu, C. Y. Ou, B. C. Shyu, and A. C. W. Huang, "Basolateral amygdala but not medial prefrontal cortex contributes to chronic fluoxetine treatments for PTSD symptoms in mice," Behavioural Neurology, vol. 2020, Article ID 8875087, 8 pages, 2020.

[30] T. Takahashi, K. Shimizu, K. Shimazaki, H. Toda, and M. Nibuya, "Environmental enrichment enhances autophagy signaling in the rat hippocampus," Brain Research, vol. 1592, pp. 113-123, 2014.

[31] C. A. Schneider, W. S. Rasband, and K. W. Eliceiri, "NIH image to ImageJ: 25 years of image analysis," Nature Methods, vol. 9, pp. 671-675, 2012.

[32] T. D. Schmittgen, "Real-time quantitative PCR," Methods, vol. 25, pp. 383-385, 2001.

[33] H. Hendriksen, B. Olivier, and R. S. Oosting, "From nonpharmacological treatments for post-traumatic stress disorder to novel therapeutic targets," European Journal of Pharmacology, vol. 732, pp. 139-158, 2014.

[34] H. Hendriksen, J. Prins, B. Olivier, and R. S. Oosting, "Environmental enrichment induces behavioral recovery and enhanced hippocampal cell proliferation in an antidepressant-resistant animal model for PTSD," PLoS One, vol. 5, article e11943, 2010.

[35] A. J. Hannan, "Environmental enrichment and brain repair: harnessing the therapeutic effects of cognitive stimulation and physical activity to enhance experience-dependent plasticity," Neuropathology and Applied Neurobiology, vol. 40, no. 1, pp. 13-25, 2014.

[36] C. Girbovan and H. Plamondon, "Environmental enrichment in female rodents: considerations in the effects on behavior and biochemical markers," Behavioural Brain Research, vol. 253, pp. 178-190, 2013.

[37] I. P. Pavlov, Conditioned reflexes, I. P. Pavlov, Ed., Oxford University Press, Oxford, UK, 1927.

[38] K. M. Fraser and P. C. Holland, "Occasion setting," Behavioral Neuroscience, vol. 133, pp. 145-175, 2019.

[39] N. A. Schmajuk, J. A. Lamoureux, and P. C. Holland, "Occasion setting: a neural network approach," Psychological Review, vol. 105, pp. 3-32, 1998.

[40] M. J. Kim, R. A. Loucks, A. L. Palmer et al., "The structural and functional connectivity of the amygdala: from normal emotion to pathological anxiety," Behavioural Brain Research, vol. 223, pp. 403-410, 2011.

[41] T. F. Giustino and S. Maren, "The role of the medial prefrontal cortex in the conditioning and extinction of fear," Frontiers in Behavioral Neuroscience, vol. 9, p. 298, 2015.

[42] M. Koenigs and J. Grafman, "Posttraumatic stress disorder: the role of medial prefrontal cortex and amygdala," The Neuroscientist, vol. 15, pp. 540-548, 2009.
[43] J. W. Rudy and R. J. Sutherland, "Configural association theory and the hippocampal formation: an appraisal and reconfiguration," Hippocampus, vol. 5, pp. 375-389, 1995.

[44] I. Izquierdo, C. R. Furini, and J. C. Myskiw, "Fear memory," Physiological Reviews, vol. 96, no. 2, pp. 695-750, 2016.

[45] S. Languille, S. Davis, P. Richer, C. Alcacer, S. Laroche, and B. Hars, "Extracellular signal-regulated kinase activation is required for consolidation and reconsolidation of memory at an early stage of ontogenesis," The European Journal of Neuroscience, vol. 30, no. 10, pp. 1923-1930, 2009.

[46] A. J. McDonald and D. D. Mott, "Functional neuroanatomy of amygdalohippocampal interconnections and their role in learning and memory," Journal of Neuroscience Research, vol. 95, pp. 797-820, 2017.

[47] A. Badiani, M. M. Oates, H. E. W. Day, S. J. Watson, H. Akil, and T. E. Robinson, "Amphetamine-induced behavior, dopamine release, and c-fos mRNA expression: modulation by environmental novelty," The Journal of Neuroscienc., vol. 18, no. 24, pp. 10579-10593, 1998.

[48] A. Badiani, M. M. Oates, H. E. W. Day, S. J. Watson, H. Akil, and T. E. Robinson, "Environmental modulation of amphetamine-induced c-fos expression in D1 versus D2 striatal neurons," Behavioural Brain Research, vol. 103, pp. 203 209, 1999.

[49] P. Holland, "Ocassion setting in Pavlovian conditioning," in The Psychology of Learning and Motivation, D. L. Medin, Ed., pp. 69-125, CA: Academic Press, San Diego, 1992. 